\title{
Éditorial \\ Anthropologie historique des violences de masse
}

\author{
Ilan $\overline{\text { Lew \& Daniel Bonnard }}$
}

Ce numéro thématique de la revue Emulations désire présenter au lecteur des contributions de jeunes chercheuses et chercheurs dont les préoccupations épistémologiques tendent à mieux comprendre la capacité des êtres humains à s'entre-détruire. Les courants scientifiques représentés sont, pour ce faire, issus de disciplines et de contextes linguistiques de production variés. Certains des travaux s'inscrivent dans des recherches en histoire culturelle de la violence de guerre, d'autres en sociologie de la violence extrême ou dans la Täterforschung allemande, d'autres encore dans le domaine de la Transitional Justice. Ces contributrices et contributeurs constituent une sorte de réseau international de jeunes chercheurs avec lequel les éditeurs, reliés tous deux à Genève, ont eu le privilège de pouvoir collaborer: il comprend le Centre d'Etudes Historiques et le Centre d'Analyse et d'Intervention Sociologiques de l'EHESS à Paris, le Center for Interdisciplinary Memory Research de Flensburg, l'International Research and Documentation Center for War Crimes Trials à Marburg en Allemagne, ainsi que l'Institut des Hautes Etudes Internationales, en Suisse.

Les recherches exposées puisent pour beaucoup leur manière d'interroger les violences collectives dans l'anthropologie, et c'est sur cet aspect qu'elles disposent de fortes relations entre elles. Si le caractère historien de ces études se justifie de par le fait qu'elles reviennent sur les violences de masse du XXème siècle, soit des éléments parmi les plus symptomatiques de cette époque qu'Eric Hobsbawm dénomme "l'âge des extrêmes", le recours à l'anthropologie est déjà présent dans le projet consistant à regrouper des études de cas attachées à une diversité de contextes. Ceci dit, c'est certainement le positionnement général dans la production de connaissances, dans la confrontation aux violences comme objet de recherche, qui donne au recueil publié son orientation sous-tendue dans l'espace des savoirs.

À ce titre, dans son ouvrage intitulé Historische Anthropologie, l'historien suisse Jakob Tanner décrit la démarche épistémologique dont il est question dans ce numéro comme «une histoire de l'être humain concret, centrée sur ses expériences, qui vise à en connaître la corporéité, les dispositions mentales et les pratiques sociales » (2004, 98, notre traduction) ou comme le dit magnifiquement Claude Lévi-Strauss en réponse à une question de Bernard Pivot sur ce qu'est et à quoi sert l'anthropologie: «Il s'agit d'essayer de comprendre l'homme par la totalité de ses expériences et de ses réalisations » (Lévi-Strauss, 1984). En disant cela, Lévi-Strauss cherchait surtout à expliquer l'intérêt qu'il portait aux sociétés amérindiennes et africaines en tant 
qu'étape ultime dans la prospection et l'éloignement pour interroger l'homme. On ne saurait nier la grande pertinence d'une telle réponse quand on la rapporte à ce mouvement de retour sur l'énigme de la violence collective dans nos sociétés, comme dans les sociétés extra-européennes, pour questionner les pratiques inhérentes au «pouvoir de détruire » (Sémelin 2005, 20).

Pour se faire une idée plus concrète des objets et des enjeux appliqués dans une telle perspective aux violences de masse, nous allons avoir recours à l'historien français Stéphane Audoin-Rouzeau dont les travaux, dans la continuité notamment de l'historien britannique John Keegan, offrent des pistes aussi productives qu'innovatrices pour questionner le fait guerrier. Stéphane Audoin-Rouzeau a eu la grande amabilité de répondre à un entretien pour ce numéro de revue dans lequel il revient non seulement sur ses écrits les plus récents, mais aussi sur ses premières monographies sur la Grande Guerre qui étudient les bouleversements des représentations et des discours dans certains secteurs de la société française en guerre. L'interview évoque son récent engagement de chercheur dans l'étude de contextes très distincts des violences de masse, en premier lieu desquels figure le génocide rwandais.

Dans son ouvrage Combattre, au caractère plus épistémologique et programmatique, il invite à étudier la violence de guerre « comme un fait social, (...) dans ses formes, dans les marques qu'elle laisse sur les corps, dans les représentations qu'elle fait émerger, dans ce qu'elle nous permet de comprendre sur les intentions de l'ennemi, la pratique de violence comme une confrontation des interprétations parmi les belligérants » $(2008,22)$.

Ce numéro thématique regroupe des objets de recherches différents, en particulier au regard des types de violences de masse étudiés. On n'y explore pas seulement les violences de guerre déjà évoquées, mais également des violences étatiques fortement asymétriques qui relèvent du meurtre de masse. Malgré les différences notoires, portant aussi bien sur les techniques de déshumanisation que sur les modes de destruction mis en œuvre, nous sommes convaincus qu'il fait sens d'étudier des violences de différents types de manière comparative dans la mesure où elles ont en commun d'être l'expression du massacre en tant que fait social, envisagé ici à l'intersection entre les pratiques et les discours de leurs auteurs. Les violences coloniales, qui entrent pleinement dans ce genre de questionnement, n'y font pas exception, même si elles ne font pas l'objet de contribution dans ce recueil.

En prenant le massacre comme point de départ, et, dans le même temps, en concevant son intrication dans l'agir social, mettant en jeu croyances et représentations, l'anthropologie livre un apport décisif à l'étude des violences. Dans cette perspective, c'est notamment la question de la signification des violences en tant que relation sociale extrême à l'égard d'un Autre, d'un ennemi, qui est posée. On part alors du présupposé que les violences disposent d'un sens pour ceux qui les ont commises, qui circule entre le caractère performatif des pratiques et leurs représentations langa- 
gières ou visuelles. On voit bien dans un travail comme celui de Juliette Courmont le premier de ce numéro - abordant notamment les pratiques humiliantes des soldats sur les civils durant la Première Guerre Mondiale, qu'il existe des enjeux de confrontation des interprétations entre le message que veut transmettre ceux qui commettent les violences et ce qu'en fait la collectivité qui la subit. De son côté, Chloé Rabanes interroge les logiques de violence qu'impliquent l'enlèvement durant la guerre civile au Liban et les significations induites par la capture des corps, entre autres en tant qu'enjeu territorial.

Des recherches récentes, issues aussi bien des travaux du sociologue allemand Harald Welzer, que de réflexions d'historiens sur la réception des violences nazies dans la société allemande post-génocidaire, portent un intérêt marqué pour les «sentiments moraux » propres aux bourreaux, tant ceux-ci semblent inséparables de l'analyse des actes perpétrés. Dans son ouvrage très remarqué Anständig geblieben (2010), l'historien Raphael Gross met en évidence les aspects constitutifs d'une « moralité » héritée de l'emprise du nazisme sur la société allemande, dans le sens d'un système de valeurs partagé, à même de produire des formes de justification et de légitimer - à l'image des efforts pour ancrer l'idéologie raciale dans le droit - la perpétration d'actes de meurtre de masse. En examinant les discours et les pratiques du registre de l'après-violence, Ilan Lew s'attache à mettre en évidence les «valeurs morales » exprimées chez d'anciens bourreaux en situation d'entretien. Friederike Mieth, quant à elle, problématise la question de la coexistence avec d'anciens auteurs de violences en montrant les conditions de réintégration d'ex-combattants en Sierra Leone.

De son côté, Brieg Capitaine recense une enquête sociologique de Claude Juin (2012), un ancien de la Guerre d'Algérie, qui vient interroger l'articulation entre le vécu subjectif et le point de vue sur les pairs quand il analyse la transformation des régiments à mesure que les atrocités commises entrent dans la pratique quotidienne des soldats.

D'autres questionnements, initiés en Allemagne par les travaux de la Täterforschung, reviennent sur les enjeux liés à l'usage et à la signification de l'espace dans les violences de masse. La gestion de l'espace, mais aussi sa production en termes de catégorie sociale donne lieu à des «espaces de la violence » - pour reprendre le titre de la contribution de Michaela Christ - qui agissent comme cadre de référence des pratiques de meurtre. Ceci est rendu tangible dans le cadre d'une étude de cas sur le meurtre des Juifs de Berdytchev, en Ukraine, pendant l'occupation allemande. La façon dont l'espace est marqué par les violences, où leurs auteurs s'emparent d'un lieu pour le remodeler profondément, est ainsi caractéristique des lieux de massacre. Il s'agit de comprendre ce qu'on fait des corps dans l'espace, comme ce qu'on fait des corps comme des espaces. Cette préoccupation marque également l'étude de cas présentée par Barbara Martin sur Babi Yar, ce ravin de la ville de Kiev, où des membres des Einsatzgruppen, aidés par la police auxiliaire 
ukrainienne, ont assassiné près de 100.000 personnes appartenant à différents groupes pendant la deuxième guerre mondiale.

L'enjeu est de mettre en perspective des formes de violences collectives et étatiques ayant fait un grand nombre de victimes, sans pour autant que celles-ci ne prennent forcément la forme du génocide. Le recours au terme de «violences de masse », introduit par le chercheur français Jacques Sémelin (2005, 2008), permet de laisser consciemment en suspens, sans la mettre de côté, la question sensible de la qualification des violences. S'il est crucial de traiter la mise en catégorie juridique comme une part inséparable des discours, en particulier ultérieurs aux violences, celle-ci ne doit pas constituer un jugement à priori, qui viendrait faire obstacle à un examen minutieux des conditions de perception et d'usage politique des violences par les acteurs eux-mêmes. Pour ne pas éluder cette question difficile, le numéro se conclut par la recension réalisée par Daniel Bonnard d'une publication récente du Fritz Bauer Institut de Francfort (2012) qui rassemble les points de vue de chercheurs contemporains discutant des enjeux relatifs à la comparaison des violences de masse.

\section{Bibliographie}

Audoin-RouZEAU, S. (2008). Combattre, Une anthropologie historique de la guerre moderne (XIXe-XXI ${ }^{\mathrm{e}}$ siècle), Paris : Seuil.

Fritz BAUER InSTITUT, STEINBACHER, S. (2012). Holocaust und Völkermorde. Die Reichweite des Vergleichs. Jahrbuch 2012 zur Geschichte und Wirkung des Holocaust.

Gross, R., (2009). Anständig geblieben. Nationalsozialistische Moral, Francfort: Fischer Verlag.

HobsBawm, E., (1999). L'Age des extrêmes: le court XX siècle (1914-1991), Bruxelles: Ed. Complexe.

JuIN, C., (2012). Des soldats tortionnaires. Guerre d'Algérie: des jeunes gens ordinaires confrontés à l'intolérable, Paris : Robert Laffont.

KeEgAN, J. (2013 [1976]). Anatomie de la bataille : Azincourt 1415, Waterloo 1815, la Somme 1916, Paris : Ed. Perrin.

LÉvi-Strauss, C. (1984). Entretien avec Bernard Pivot du 4 mai 1984, France: INA, 72'.

SEMElin, J. (2005). Purifier et détruire. Usages politiques des massacres et génocides, Paris: Seuil. 
SEMELIN, J. (2008). « Notre approche scientifique » in Online Encyclopedia of Mass Violence, Paris : CERI-Science Po.

URL : http://www.massviolence.org/Notre-approche-scientifique (consulté le 14 septembre 2013).

TANNER, J. (2004). Historische Anthropologie zur Einführung, Hamburg: Junius Verlag. 